Revue d'histoire de l'enfance « irrégulière »

Le Temps de l'histoire

6 | 2004

Les sciences du psychisme et l'enfance « irrégulière »

\title{
Victor Girard : de la psychiatrie à la prévention spécialisée
}

Jacques Bourquin

\section{(2) OpenEdition \\ Journals}

Édition électronique

URL : http://journals.openedition.org/rhei/842

DOI : $10.4000 /$ rhei.842

ISBN : 978-2-7535-1644-1

ISSN : 1777-540X

Éditeur

Presses universitaires de Rennes

Édition imprimée

Date de publication : 15 novembre 2004

Pagination : 253-254

ISSN : 1287-2431

Référence électronique

Jacques Bourquin, "Victor Girard : de la psychiatrie à la prévention spécialisée », Revue d'histoire de l'enfance « irrégulière » [En ligne], 6 | 2004, mis en ligne le 31 mai 2007, consulté le 03 décembre 2020 URL : http://journals.openedition.org/rhei/842 ; DOI : https://doi.org/10.4000/rhei.842 
Michèle Becquemin

Protection de l'enfance. L'action de l'association Olga Spitzer,

1923-2003

Préface de Michel Chauvière

Erès, Ramonville-Saint-Agne, 2003, 255 p.

Voici un livre qui marquera dans la connaissance de la naissance du travail social. Michèle Becquemin, dans un beau travail qui repose sur une étude d'archives extrêmement fouillée, nous restitue l'histoire d'une association qui, depuis 80 ans, est au cœur même de la "fabrique du social ", pour reprendre l'expression de Michel Chauvière dans sa préface.

Elle nous fait découvrir combien le Service social de l'enfance en danger moral (SSEDM), devenu plus tard l'association Olga Spitzer, fut un véritable laboratoire d'expériences qui a initié tout une série de secteurs d'intervention dans le domaine de la protection de l'enfance. Le SSEDM apparaît comme une véritable matrice ; Michèle Becquemin emploie le beau terme d'" arborescence ".

Créé en 1923, le SSEDM s'inscrit, en référence de la loi de 1912 sur l'enfance délinquante, comme un outil indispensable à l'application de ce texte dans un contexte, celui de l'après-guerre, où « la crise démographique alarmante, l'affaiblissement moral et physique des populations, les préoccupations familialistes et natalistes " amènent à se préoccuper de l'enfance en danger moral.

La création du SSEDM est fortement liée à la personnalité de ses promoteurs, sur lesquels Michèle Becquemin nous apporte des éléments très précieux. Certains sont déjà connus, comme Henri Rollet qui, dès 1890, milite dans les comités de défense des enfants traduits en justice, où il développe l'idée d'enfance en danger moral. Il sera le premier juge des enfants de la loi de 1912. D'autres apparaissent après la guerre. C'est une américaine, Chloé Owings, arrivée en France en 1916 avec une pratique de travail social à New-York, qui sera avec Henri Rollet l'instigatrice du SSEDM. La thèse de sociologie qu'elle soutient à la Sorbonne en 1923, sur le travail social, contribuera largement à renforcer la légitimi-

Jacques Bourquin / p. 289 à 292 
té du service dans le monde intellectuel. Une autre américaine, Olga Spitzer, fera bénéficier le SSEDM, pendant plus de 20 ans, d'un considérable soutien financier lié à la fortune de son mari, avant que l'État ne devienne le pourvoyeur principal de l'institution. Le rôle d'Olga Spitzer ne se limitera pas à cet aspect financier ; elle défendra la cause de l'enfance en danger moral dans de multiples organismes.

D’autres personnages sont très liés à ces promoteurs : Th. Vieillot, une assistante sociale qui sera la première directrice du SSEDM, des médecins, dont le docteur Simon qui fut le collaborateur d'Alfred Binet, une avocate, Hélène Campinchi, qui sera une des rédactrices de l'ordonnance de 1945 sur l'enfance délinquante, des politiques, souvent issus de la mouvance radicale socialiste, que l'on retrouvera dans le conseil d'administration. Le SSEDM sera reconnu d'utilité publique dès 1928.

En fait, l'Etat, cantonné à l'assistance et à l'administration pénitentiaire, reste très en retrait. Il fait assurer par le secteur privé tout le champ d'expérimentation en application de la loi de 1912.

L'objectif du SSEDM - et cela s'inscrit pleinement dans le combat mené par Henri Rollet depuis la fin du XIXème siècle dans le domaine de l'enfance en danger moral - est de faire reculer la référence à la sanction, aussi bien dans le domaine civil (la correction paternelle) que dans celui du pénal (la délinquance juvénile), en valorisant, en développant la dimension protectrice et éducative.

Dans une deuxième partie, intitulée " Développement associatif et politiques sociales », Michèle Becquemin expose les grandes étapes de la croissance du SSEDM en référence au contexte politique et social. Elle souligne cette capacité que l'institution a, dans les années trente, d'interpeller le champ politique, son influence au moment des décrets-lois d'octobre 1935 sur l'assistance éducative et la dépénalisation du vagabondage dans un souci de "globalisation " de la protection civile des mineurs. L'application du décret sera hélas décevante par manque de moyens. À la lecture de cette partie, on perçoit les aléas que rencontre le SSEDM à la veille de la guerre, mais aussi toutes les stratégies développées qui contribueront progressivement à mettre en place une politique de l'enfance inadaptée et à l'évolution des décrets de 1935 vers l'ordonnance du 23 décembre 1958 sur l'en- 
fance en danger, sans oublier l'ordonnance du 2 février 1945 qui officialise la notion d'éducabilité du mineur en danger.

Au lendemain de la guerre, le SSEDM entame une période d'expansion : il y avait cinquante salariés en 1939, deux cent trente en 1971, près de neuf cents aujourd'hui. Cette longue histoire, le développement progressif de l'institution ne fut pas sans incidence sur l'histoire des professions du secteur de l'enfance inadaptée. Le SSEDM fut un creuset de l'apparition des assistantes sociales. C'est cet aspect que Michèle Becquemin aborde dans sa troisième partie.

Le recours important au bénévolat durera jusqu'à la fin de la dernière guerre, mais, déjà dans les années vingt, le SSEDM fut un haut lieu de professionnalisation, en particulier avec le recours aux premières écoles de service social dont l'École pratique de service social à Paris, où Chloé Owings enseigne. À l'avant-garde du social, le SSEDM fut vite confronté à des problèmes de recrutement et de formation.

Bien que l'École pratique de service social soit mixte, il n'en sera pas de même pour la profession qui se crée. Le SSEDM sera longtemps une organisation de femmes qui travaille dans un monde d'hommes, celui des magistrats et des médecins.

Michèle Becquemin écrit quelques pages savoureuses sur cet ordre féminin, qu'elle illustre de témoignages. Il faudra attendre le début des années soixante, l'application de l'ordonnance de 1958 et le développement de l'AEMO, pour que l'on voit arriver, avec les éducateurs, les premiers hommes dans l'institution. Ceux-ci resteront toutefois minoritaires jusqu'au début des années quatre-vingts ; on recrutera surtout des éducatrices et l'on évoquera, il y a encore 20 ans, "la lignée matriarcale d'un bout à l'autre de la chaîne du SSE ", de l'assistante à l'éducatrice.

La dernière partie du livre est consacrée à l'établissement phare du SSEDM, le foyer de Soulins qui, dès 1928, inscrit l'importance de l'observation dans l'intervention auprès de l'enfance irrégulière et inadaptée. Soulins préfigure les premiers centres d'observation de la guerre et de l'après-guerre.

Un livre passionnant, d'une haute tenue intellectuelle, qui pose de multiples questions, en particulier aujourd'hui où la tendance serait de 
revenir à des conceptions plus punitives et moins centrées sur le jeune. La notion d'enfant en danger s'estompe pour laisser la place d'un côté à l'enfant maltraité victime, de l'autre à l'enfant délinquant coupable.

Jacques Bourquin 